\section{Nontuberculous Mycobacterial Pulmonary Disease from Mycobacterium hassiacum, Austria}

\author{
Helmut J.F. Salzer, Bakari Chitechi, Doris Hillemann, \\ Michael Mandl, Christian Paar, Monika Mitterhumer, \\ Bernd Lamprecht, Florian P. Maurer
}

Author affiliations: Kepler University Hospital, Linz, Austria (H.J.F. Salzer, B. Chitechi, C. Paar, M. Mitterhumer, M. Mandl, B. Lamprecht); National and WHO Supranational Reference Centre for Mycobacteria-Borstel, Borstel, Germany (D. Hillemann, F.P. Maurer)

DOI: https://doi.org/10.3201/eid2611.191718

The clinical relevance of newly described nontuberculous mycobacteria is often unclear. We report a case of pulmonary infection caused by Mycobacterium hassiacum in an immunocompetent patient in Austria who had chronic obstructive pulmonary disease. Antimicrobial drug susceptibility testing showed low MICs for macrolides, aminoglycosides, fluoroquinolones, tetracyclines, imipenem, and linezolid.

urrently, $>170$ species of nontuberculous mycobacteria (NTM) are recognized (1), most considered nonpathogenic to humans. However, some NTM can cause severe pulmonary disease. We recently observed a case of NTM pulmonary disease (NTM-PD) in Austria that was caused by Mycobacterium hassiacum.
In January 2019, a 62-year-old man was admitted to the outpatient clinic at Kepler University Hospital in Linz, Austria, having had dry cough and progressive dyspnea for several months. No weight loss or night sweats were reported. He had a medical history of chronic obstructive pulmonary disease with severe emphysema because of cigarette smoking. The patient had no history of tuberculosis or NTM-PD and was unaware of any contact with persons with mycobacterial infections.

Chest radiograph showed new consolidations in the right and left upper lung lobes compared with images obtained 1 year before. We performed a high-resolution computed tomography scan of the chest and an 18F-fluorodeoxyglucose positron emission tomography (18F-FDG PET) scan that indicated metabolic activity consistent with an inflammatory process (Figure). In addition, results of tests for serum lung cancer biomarkers, including CA 19-9, CEA (carcinoembryonic antigen), CYFRA 21-1, and NSE (neuron-specific enolase), and for HIV-1 and HIV-2 were negative. Transbronchial catheter aspiration from the posterior lung segment of the right upper lung lobe showed no microscopic evidence of bacteria, acid-fast bacilli, or fungi. The result from a PCR assay (Mycobacterium tuberculosis PCR kit; Geneproof, https://www.geneproof.com) for $M$. tuberculosis complex DNA was negative. Results from bacterial and fungal cultures were unremarkable. Because the patient was at high risk for developing pneumothorax due to severe emphysema, no transbronchial biopsy was taken during initial bronchoscopy.
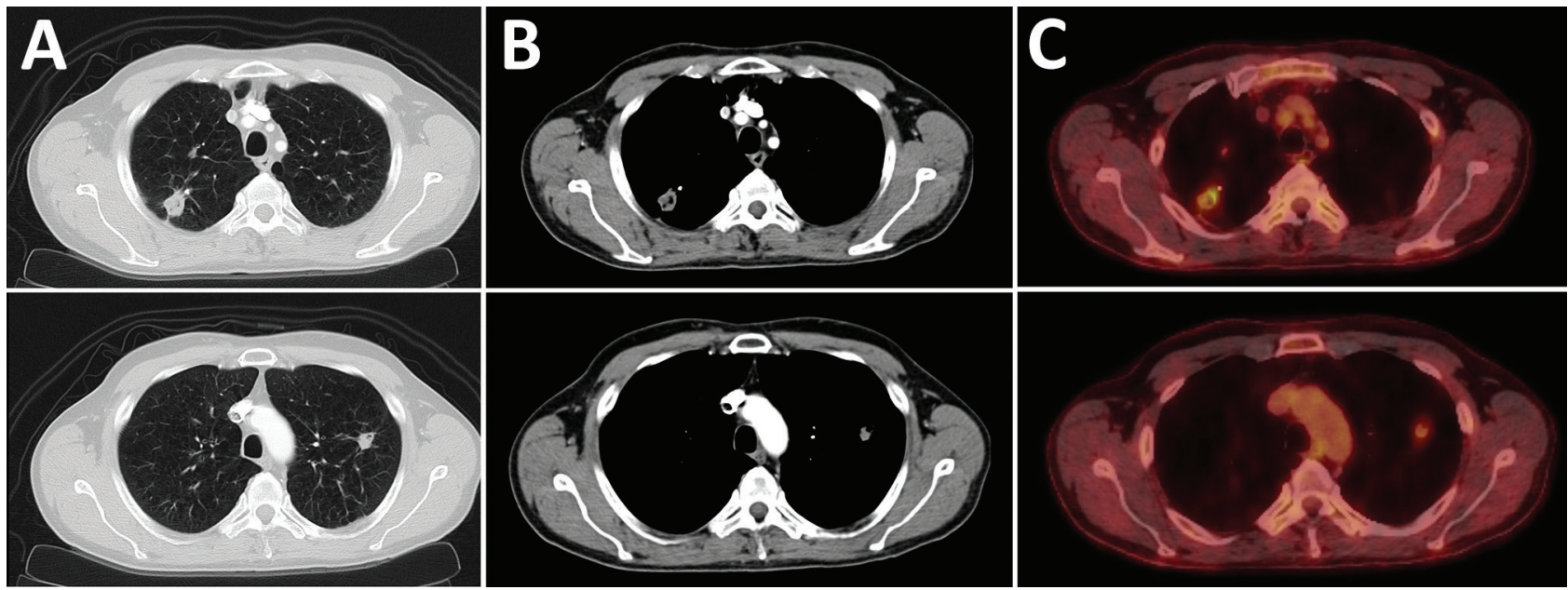

Figure. High-resolution computed tomography and 18F-fluorodeoxyglucose positron emission tomography scans of the chest showing pulmonary lesions caused by Mycobacterium hassiacum in a 62-year-old man, Austria. A and B) Computed tomography scans of the chest showing a subpleural thick-walled cavitary lesion in the posterior segment of the right upper lung lobe with associated pleural thickening and a smaller adjacent partly calcified solitary nodule. Another solid nodule of $13 \mathrm{~mm}$ diameter was found in the left upper lung lobe. C) Positron emission tomography scan showing a tracer uptake in both lesions with a standardized uptake values of 5 (top image) and 1.9 (bottom image). 
Mycobacterial culture results of the transbronchial catheter aspirate were negative after 8 weeks of incubation. Consequently, we performed a computed tomography-guided needle biopsy of the cavitary lesion in the right upper lung lobe. Histological results showed granulomatous inflammation with focal eosinophilic necrosis. We detected no atypical cells, acid-fast bacilli, or fungal hyphae and subsequent immunohistochemistry testing revealed no evidence of malignant disease.

Results of a PCR of formalin-fixed, paraffin-embedded lung tissue revealed no DNA of $M$. tuberculosis (MTB Nested ACE Detection Kit, Seegene, http:/ / www.seegene.com) or of 13 different NTM species (MYCO Direct 1.7 LCD-Array Kit; Chipron, https:/ / www.chipron.com). However, native lung tissue was used to set up both liquid (MGIT 960 system, Becton Dickinson, https:/ / www.bd.com) and solid mycobacterial cultures (Löwenstein-Jensen and Middlebrook agar), which showed growth after 21 days of incubation at $37^{\circ} \mathrm{C}$. $16 \mathrm{~S}$ rRNA gene sequence analysis identified M. hassiacum (GenBank accession no. AF547933.1).

We analyzed the samples by DNA sequencing of the first $500 \mathrm{bp}$ of the $5^{\prime}$ region of the $16 \mathrm{~S}$ rRNA gene $(2,3)$. The analyzed DNA fragment showed 500-bp identity with the M. hassiacum type strain $384916 S$ ribosomal RNA (sequence identification NR_026011.1). More than 25 mismatches were found with the type strains of $M$. thermoresistibile strain NCTC10409, $M$. goodii strain ATCC 700504, and M. celeriflavum strain AFPC-000207. Antimicrobial susceptibility testing by broth microdilution method according to Clinical and Laboratory Standards Institute guidelines $(4,5)$ showed low MICs for clarithromycin $(\leq 0.06 \mu \mathrm{g} / \mathrm{mL})$, amikacin $(\leq 1 \mu \mathrm{g} / \mathrm{mL})$, tobramycin $(\leq 1 \mu \mathrm{g} / \mathrm{mL})$, linezolid $(\leq 1.0$ $\mu \mathrm{g} / \mathrm{mL})$, moxifloxacin $(\leq 0.25 \mu \mathrm{g} / \mathrm{mL})$, ciprofloxacin $(0.25 \mu \mathrm{g} / \mathrm{mL})$, doxycycline $(\leq 0.12 \mu \mathrm{g} / \mathrm{mL})$, minocycline $(\leq 1 \mu \mathrm{g} / \mathrm{mL})$, tigecycline $(0.25 \mu \mathrm{g} / \mathrm{mL})$, imipenem $(\leq 2.0 \mu \mathrm{g} / \mathrm{mL})$, and trimethoprim/sulfamethoxazole $(4.75 \mu \mathrm{g} / \mathrm{mL})(4,5)$. Comparably high MIC values were observed for cefoxitin $(32 \mu \mathrm{g} / \mathrm{mL})$, ceftriaxone $(>64 \mu \mathrm{g} / \mathrm{mL})$, and cefepime $(>32 \mu \mathrm{g} / \mathrm{mL})$. Antimycobacterial treatment, including clarithromycin (500 mg orally $2 \times / \mathrm{d})$, moxifloxacin $(400 \mathrm{mg}$ orally $1 \times / \mathrm{d})$, and minocycline (100 mg orally $2 \times / \mathrm{d})$, was initiated. On first follow-up visit, the patient indicated that the treatment had been well tolerated.

Since M. hassiacum was described as a new species in 1997, 3 cases of suspected infections caused by $M$. hassiacum have been reported in the medical literature (6). So far, M. hassiacum has been reported as the causative agent for peritonitis and cystitis $(7,8)$. In addition, M. hassiacum was recently isolated from a respiratory sample in a patient in Germany with exacerbation of chronic obstructive pulmonary disease (9). However, that patient likely did not have NTM-PD because $M$. hassiacum was isolated in only 1 of 3 sputum samples, he showed no NTM-specific radiological abnormalities in a chest radiograph, and his clinical condition improved rapidly without any antimycobacterial treatment.

In contrast, the patient we report fulfills 3 diagnostic criteria of NTM-PD: 1) an NTM-specific radiologic pattern characterized by new nodules in both upper lung lobes in a high-resolution computed tomography scan of the chest, 2) a positive culture result from a sterile computed tomography-guided needle biopsy, as well as typical mycobacterial histopathologic features including granulomatous inflammation, and 3) exclusion of other disorders including pulmonary tuberculosis or lung cancer (10).

In conclusion, detection of M. hassiacum in patients fulfilling the criteria for NTM-PD should be considered a potentially relevant finding. Further studies are needed to evaluate the precise role of $M$. hassiacum as an emerging mycobacterial pathogen.

\section{Acknowledgments}

We thank the patient for giving permission to publish the case.

\section{About the Author}

Dr. Salzer works as a senior physician in the Department of Pulmonary Medicine at the Kepler University Hospital in Linz, Austria. His primary research interests include chronic pulmonary infections, especially nontuberculous mycobacterial pulmonary diseases and chronic pulmonary aspergillosis.

\section{References}

1. Fedrizzi T, Meehan CJ, Grottola A, Giacobazzi E, Fregni Serpini G, Tagliazucchi S, et al. Genomic characterization of nontuberculous mycobacteria. Sci Rep. 2017;7:45258. https://doi.org/10.1038/srep45258

2. Richter E, Niemann S, Gloeckner FO, Pfyffer GE, Rüsch-Gerdes S. Mycobacterium holsaticum sp. nov. Int J Syst Evol Microbiol. 2002;52:1991-6. https:/ / doi.org/ 10.1099/00207713-52-6-1991

3. Böddinghaus B, Rogall T, Flohr T, Blöcker H, Böttger EC. Detection and identification of mycobacteria by amplification of rRNA. J Clin Microbiol. 1990;28:1751-9. https://doi.org/10.1128/JCM.28.8.1751-1759.1990

4. Clinical and Laboratory Standards Institute. Susceptibility testing of mycobacteria, Nocardia spp., and other aerobic actinomycetes, 3rd edition (standard M24). Wayne (PA): The Institute; 2018.

5. Clinical and Laboratory Standards Institute. Performance standards for susceptibility testing of mycobacteria, Nocardia 
spp., and other aerobic actinomycetes, 3rd edition (standard M62). Wayne (PA): The Institute; 2018.

6. Schröder KH, Naumann L, Kroppenstedt RM, Reischl U. Mycobacterium hassiacum sp. nov., a new rapidly growing thermophilic mycobacterium. Int J Syst Bacteriol. 1997; 47:86-91. https://doi.org/10.1099/00207713-47-1-86

7. Jiang $\mathrm{SH}$, Roberts DM, Clayton PA, Jardine M. Non-tuberculous mycobacterial PD peritonitis in Australia. Int Urol Nephrol. 2013;45:1423-8. https:// doi.org/10.1007/ s11255-012-0328-4

8. Tortoli E, Reischl U, Besozzi G, Emler S. Characterization of an isolate belonging to the newly described species Mycobacterium hassiacum. Diagn Microbiol Infect Dis. 1998;30:193-6. https://doi.org/10.1016/S07328893(97)00242-3

9. Deinhardt-Emmer S, Höring S, Mura C, Hillemann D, Hermann B, Sachse S, et al. First time isolation of Mycobacterium hassiacum from a respiratory sample. Clin Med Insights Circ Respir Pulm Med. 2018;12:1179548417747529. https://doi.org/10.1177/1179548417747529

10. Griffith DE, Aksamit T, Brown-Elliott BA, Catanzaro A, Daley C, Gordin F, et al.; ATS Mycobacterial Diseases Subcommittee. An official ATS/IDSA statement: diagnosis, treatment, and prevention of nontuberculous mycobacterial diseases. Am J Respir Crit Care Med. 2007;175:367-416. Erratum in Am J Respir Crit Care Med. 2007 Apr 1;175:744-5. https:/ / doi.org/10.1164/rccm.200604-571ST

Address for correspondence: Helmut J.F. Salzer, Department of Pulmonary Medicine, Kepler University Hospital, Krankenhausstrasse 9, 4021 Linz, Austria; email: helmut.salzer@ kepleruniklinikum.at

\section{Large Outbreak of Guillain- Barré Syndrome, Peru, 2019}

\author{
César V. Munayco, Ronnie G. Gavilan, \\ Gladys Ramirez, Manuel Loayza, Maria L. Miraval, \\ Erin Whitehouse, Radhika Gharpure, Jesus Soares, \\ Hans Vasquez Soplopuco, James Sejvar
}

Author affiliations: Centro Nacional de Epidemiología Prevención y Control de Enfermedades, Lima, Peru (C.V. Munayco, G. Ramirez, M. Loayza); Instituto Nacional de Salud, Lima (R.G. Gavilan, M.L. Miraval, H.V. Soplopuco); Centers for Disease Control and Prevention, Atlanta, Georgia, USA (E. Whitehouse, R. Gharpure, J. Soares, J. Sejvar)

DOI: https://doi.org/10.3201/eid2611.200127
Outbreaks of Guillain-Barré syndrome (GBS) are uncommon. In May 2019, national surveillance in Peru detected an increase in GBS cases in excess of the expected incidence of 1.2 cases $/ 100,000$ population. Several clinical and epidemiologic findings call into question the suggested association between this GBS outbreak and Campylobacter.

Cuillain-Barré syndrome (GBS) is the most com$\exists$ mon form of acute flaccid paralysis worldwide (1). It is characterized by motor weakness, areflexia, sensory abnormalities, and cytoalbuminologic dissociation in cerebrospinal fluid (2). An upper respiratory or gastrointestinal illness typically precedes GBS (3). Campylobacter jejuni infection is the most frequently identified precipitant of GBS and usually is associated with the acute motor axonal neuropathy form of GBS (4).

During the week of May 26, 2019, the Peruvian Ministry of Health surveillance system detected several cases of suspected GBS that exceeded the expected incidence of 1.2 cases/100,000 persons/year (i.e., 29 cases/year) (1). Since 2016, hospitals in Peru have reported suspected GBS cases to a passive surveillance system (https://www.dge.gob.pe/portal/index. php?option $=$ com_content\&view $=$ article\&id $=653$ ). In early May 2019, when the system was modified to an active surveillance system because of increasing incidence, the National Center of Epidemiology, Prevention, and Disease Control solicited cases. Examining physicians classified cases in accordance with the Brighton Collaboration case definition for GBS (5). The Instituto Nacional de Salud tested serum, urine, nasal swab samples, and feces for infectious pathogens using molecular panels for multipathogen detection (bioMérieux, https:/ / www.biomerieux-diagnostics.com) and conventional microbiology assays.

During May 20-July 27, 2019, we identified 683 suspected or confirmed GBS cases in Peru. The largest outbreaks of GBS have involved $\approx 30-50$ cases, except for large GBS outbreaks associated with Zika virus infection; thus, this outbreak was extremely unusual because of its size. Of the cases, 32 (6.9\%) were Brighton level 1, 188 (27.5\%) were Brighton level 2, and 463 $(67.7 \%)$ were Brighton level 3. We classified Brighton levels 1 and 2 cases as confirmed, and Brighton level 3 cases as suspected (Figure; Appendix Figures 1, 2, https://wwwnc.cdc.gov/EID/article/26/11/200127-App1.pdf). Nine of Peru's 24 departments reported GBS cases, which resulted in an annualized incidence of 30.9 cases/100,000 persons/year (Table).

Of the 683 GBS patients, $287(42.0 \%)$ had descending muscle weakness and $446(65.3 \%)$ had ascending muscle weakness. Of 530 patients for whom data on 